\title{
Developing a new authenticity rating system on architectural conservation
}

\author{
M. Ulukan \& H. Arslan \\ Faculty of Architecture, Istanbul Technical University, Turkey
}

\begin{abstract}
Authenticity is one of the main phenomena of architectural conservation. At first glance it refers to the emphasizing of fidelity, originality and sincerity to the origin of historic buildings, sites or objects. This notion is a common subject of argument in the interpretation of historic buildings and sites which involves experts of the architectural conservation council. Cognoscenti are bound to decide for the difficult resolution of authenticities of historic fabrics relating to their knowledge and experience. The question about the authenticity of a historic fabric can be answered whether it is authentic or not, but this does not give an idea about the level that is "how much". The answer relies on the experts" perceptions and while one may say "very good, authentic" the others may say "not authentic" for the same historic building. This shows a lack of clarity and certainty. In this study, a new ranking system has been developed to interpret the unequal results of deterioration of historic buildings and their authenticity levels by the use of "fuzzy logic" sets. A fuzzy logic model evaluates historic buildings according to the question of "how much". Although numerical categorization sets forward sharp and mutually exclusive classes according to classical Aristotelian logic, "fuzzy logic" includes mutually inclusive classes. Fuzzy logic operators provide a formal method of manipulating linguistic variables. The usage of fuzzy logic in this system provides a comparative analysis of different conservation states of historic buildings to go through and accurate restoration works according to authenticity ratings. This study proposes objective decisions that can be interrogated and reconsidered on the authenticity of a monument rating model to evaluate the degree of authenticity of historic buildings and sites. This logical fuzzy model provides an easy tool to take reliable results. The model was briefly explained with working principles and formulations through advantageous and disadvantageous aspects.
\end{abstract}

Keywords: authenticity, cultural heritage conservation, restoration, fuzzy logic. 


\section{Introduction}

Results of scientific studies refer to a level of precision. Inferences obtained through the result of an experiment, an observation or a judgment. That can only continue on its way from hypothesis to a law by the validity in wide applications. While it is possible to move laws in sciences which have mathematical and engineering infrastructures, on the other hand, it can be pointed out that it is complicated and difficult to achieve the same goals in social sciences. In addition, these achieved final judgments and results may vary within the context of time and space. Some assumptions and reductions based on the phrase "under normal circumstances" never occur in nature in the same way. We cannot find a triangle that we are using in geometry in nature [1]. However, Aristotelian logic, used in all scientific models and accepted as valid for a long period of time in science and philosophy, is based on final acceptances of premises and conclusion. This model depends on an understanding of the duality of reality such as 0-1, yes-no, black-white as in two-valued logic. Fuzzy logic is a form of many-valued logic; it deals with reasoning that is approximate rather than fixed and exact. In contrast to Aristotelian traditional logic theory, where binary sets have two-valued logic like true or false, fuzzy logic variables may have a truth value that ranges in degree between 0 and 1 that meets the requirements of social sciences. Fuzzy logic began with the 1965 proposal of fuzzy set theory by Lutfu Askerzade [2]. However, up to now it has been generally applied in many engineering fields and is used very rarely in social sciences. The concept was hardly criticized in western world and compared with statistical and probability calculations but has started to be used especially in industrialized countries such as Japan, Singapore and Korea after the 1980s [3]. The studies with fuzzy logic have increased especially after its success in technological tools such as airconditioners, washing machines, lifts and vacuum cleaners. Fuzzy logic is used directly in nearly all engineering fields, computer technologies and the verbalization of databases.

\section{Authenticity on architectural conservation}

The word authenticity includes meanings in English, German and French such as the "original, authentic, authority, legally valid, implausible beliefs, firsthand, copied, true, do not imitate, represents the first author or source". Authenticity refers to the truthfulness of origins, attributions, commitments, sincerity, devotion and intentions" [4]. The origin of the word "authenticity" is Latin, derived from authentique. To date, its meaning has slightly changed by semantic shift to meaning "different, exotic, romantic ambient, historic parts, etc." In architectural conservation, the term authenticity was firstly seen in the Venice Charter. In the introduction part it is emphasized that "...The common responsibility to safeguard them (historic monuments) for future generations is recognized. It is our duty to hand them on in the full richness of their authenticity" [5]. In 1972 at another international conservation convention it was mentioned that authenticity of monuments for world heritage list nomination [6]. 
Another development of authenticity is "the test of authenticity" term which was included in the Operational Guidelines for the Implementation of the World Heritage Convention in 1977. Hereafter, authenticity became a unique notion of architectural conservation. Afterwards, many charters were launched relating to authenticity but the most important and overall authenticity charter is Nara, Japan in 1994. 138 expert delegates signed a final declaration which consists of 13 articles in which authenticity components were submitted as follows:

1 -form and design,

2-materials and substance,

3-use and function,

4-traditions and techniques,

5-location and setting [7].

Spirit and feeling and other internal and external factors were included to authenticity criteria. However, the first 5 criteria were generally accepted in architectural conservation. Related to these 5 criteria, historical buildings were qualified, buildings that do not exactly meet the 5 criteria were named "authentic", others remained "not authentic, but historic buildings". Finally, "authentic buildings" are candidates to the world heritage list [8] while others cannot apply for nomination.

\section{Fuzzy logic and architectural conservation}

Architectural conservation is a science and completely different area in the intersection of abstract and concrete concepts that use technological tools and machines. While final judgments can be explained by classical logic, if a concrete object-relational modelling is needed to make different decisions and approaches to fuzzy logic can be more appropriate. For example, persons who are experts in the field of the history of architecture that addresses the historical structures related to their work may not need fuzzy logic for these structures.

There is little to do in decision-making for uncertainty of a new method which does not require more information and documentary. It is very difficult for other experts of the history of architecture to identify a historic building as non-Gothic. Contrarily, there is more uncertainty (fuzzy) in the field of restoration. For example, it is very difficult to express the level of conservation of a historic building with numbers. It can be visible that the building had construction materials, form, function and a location in the settlement but how can we assume the level of conservation or what is the level of authenticity? The answer to this question is very important in the field of architectural conservation because all the implementations and interventions are formed according to these answers. Some criteria were defined and determined such as registered in class 1 , registered in class 2 and registered in class 3. However, there are many uncertainties and fuzzy parts in these criteria.

The decision making process about historic sites and buildings is also very difficult. There are many principles, laws and special cases for each building needing to be considered. 
It is quite difficult to implement the same decision making process for another building. The decision maker's knowledge, cultural experiences, reconciling independent and irregular threads, observation methods and giving decisions according to the protection of universal values are very important assets. This process needs a more fuzzy logic rather than Aristotelian logic.

The status in theories of architectural conservation is verbal. For example; we say "this building is authentique" or alternatively the option "not authentique". In classical logic there is no other option. However, during implementation there is gradualist in statements such as "little authentique", "well conserved", "good authentique". These little, well and good levels change according to person. These levels are verbal and did not state in the literature "numerically" such as $10 \%$ authenticity degree or $90 \%$ authenticity degree. This case is common not only in authenticity levels but also the level of conservation of the building and registered class of the building. According to the World Heritage List defined by the UNESCO in 2009, there were 890 works in 186 countries which 689 of them are cultural, 176 of them were natural and 25of them were mixed types [8]. If we consider all architecture heritages in whole world this can be seen as a very small number. Only in Turkey there are thousands of other heritage samples. The evaluation of these works processed through national and local decision making mechanisms. There is a lack of expertise in this levels which cause a lower level standard compared to international level. In the case of Turkey, many works of civil architecture have been demolished. The authenticity test gives the opportunity to list the buildings and "make an authenticity rating" in order to take the rest into the list. According to this model that can be a slightly difficult to implement in the first place decision-making and implementation processes all over the world can be brought as a whole on the basis of determinations made for structures

The classical method defines only 890 samples as "totally authentic" and is only $1 \%$ of the total of the historical buildings. Developed methodology within the scope of work on the authenticity of architectural modelling with the fuzzy method can be applied in many fields of architectural conservation. This model also gives us the opportunity to make a decision model for the building that is to be registered or not. In addition, the model contributes and shortens the working time and increases the quality of the buildings. Thus the study proposes a fuzzy logic modeling that enables the experts to determine the authenticity level of the buildings and discover the less original abandoned buildings for which there is no interest.

\subsection{Working principles of fuzzy logic}

It has been suggested that the logic of Aristotle has two inference values such as 0 and 1 . The positive expression fully "yes" means 1 whereas the negative 0 means "no". In other words, water verbally can be expressed as hot or cold. There were no mid values. Another sample can be a human is long or short; there were no mid values or answers according to Aristotelian logic for such questions as "how long?" or "how hot?". In fuzzy logic there is no certainty. Everything expressed mathematically is between 0 and 1 [9]. For example, water can be very 
hot, warm, slightly warm and almost warm. A human can be very long, a little bit longer, a little bit shorter or very short. These definitions are very common in our daily lives. So, fuzzy logic defines our verbal terms into mathematical models and expressions (Figures 1 and 2).

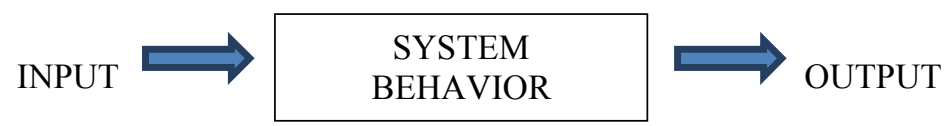

Figure 1: Classical logic system behavior.

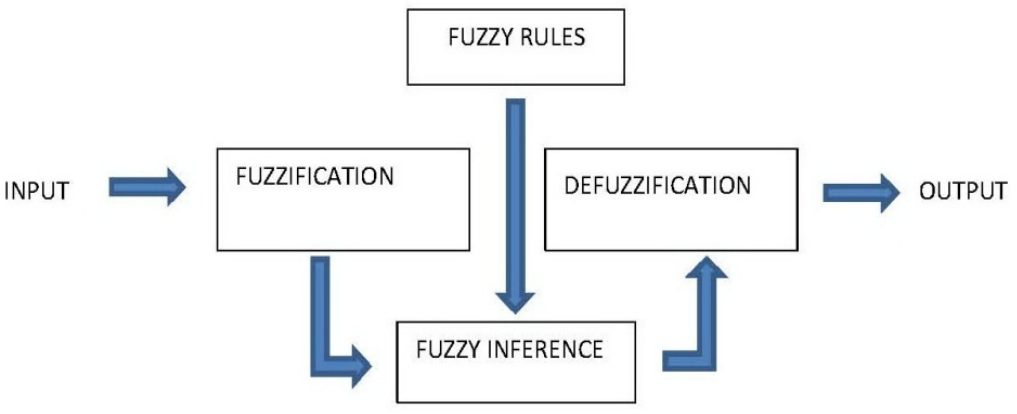

Figure 2: $\quad$ Fuzzy output working principal [1].

We often encounter the same uncertainties in our daily lives. For example, the expression "today it is very hot" may imply $25-35^{\circ} \mathrm{C}$ for one while it is $30-35^{\circ} \mathrm{C}$ for the other (Figure 3).

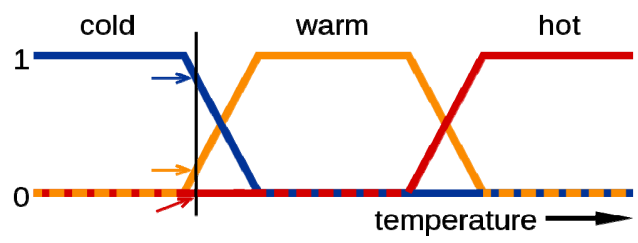

Figure 3: Linguistic variables hot [10]. (See online for color version of this figure.)

In Figure 3 the cold variable (blue) line starts from $0^{\circ} \mathrm{C}$ and continue to $13^{\circ} \mathrm{C}$ but between $0^{\circ} \mathrm{C}$ and $8^{\circ} \mathrm{C}$ it is exactly cold. From $8^{\circ} \mathrm{C}$ to $13^{\circ} \mathrm{C}$ its value is decreasing on the cold variable, but at that same range, the warm variable is increasing. For some people $9^{\circ} \mathrm{C}$ is cold, while for others it is warm. In the fuzzy logic system $10^{\circ} \mathrm{C}$, according to Figure 3 at the point of intersection of the cold (blue) and warm (yellow) line, the membership degree of cold is 0,5 and degree of warm is 0,5 . What does it mean that $10^{\circ} \mathrm{C}$ is cold at the same time it is warm. Fuzzy logic sets make this possible to be hot with a numerical variable. Its value 
is 0,5 in the fuzzy system while in the classical methods it would be only cold or only warm and the value would be 0 or 1 . Therefore, in the classical Aristotelian logic system there is no place for uncertainty, it works only with certain values. As a result, the classic system remains inadequate about uncertainty systems.

In the fuzzy system, the degree of membership is between 0 and $1(0<\mathrm{md}<1)$.

There are a lot of advantages since the membership degree is between 0 and 1. It enables us to evaluate interval uncertain values. For instance, with an air conditioner, which is controlled by a fuzzy logic micro control system the surrounding air can be controlled in comfortable situations. While the climate system can adjust the medium temperature at the same time, it can adjust humidity, fan speed etc... to provide the most comfortable condition.

Due to fact that the many advantages of the fuzzy logical system, in architectural conservation this model has been selected. Especially in the case of more than one data input fuzzy system, it is best to evaluate and to reach the most trustworthy and correct outputs. Similar to uncertain circumstances, authenticity has 5 components and it is difficult to make any decision relating to the 5-component situation. For example, "If the material is authentic and form is authentic, technique is not authentic, function is authentic and situation in a city context is medium degree authentic; the building's authenticity degree is very authentic" can be said by experts. However, material authenticity is 0,45 , form authenticity is 0,32 , technique is 0,69 , function is 0,56 , situation is 0,25 then the building's authenticity is how much? It is difficult to say anything directly looking at those numbers. Using this model it is possible. (See Table 1 and Figures 1 to 4 )

These tables were sent to specialists who were asked to assess the degree of authenticity according to the criteria in Table 1 . Such an evaluation method of fuzzy logic method corresponds with heuristic and expert opinion to the polls in statistics. The fuzzy system does not poll as well as other statistical methods. Expert opinion and the intuition of people is enough to create a logical database. Similar studies in architecture use statistical evaluations and correlation techniques which are less successful than fuzzy logic. Thus, a classical methodologies goal is based on finding a relationship between models. Those models which ignore some uncertainties are basically behavioural and dependent on cases [1]. The results obtained with the fuzzy logic system models share a tolerance to fault. According to this model created in the field of architectural conservation, a working expert's personal opinion and human error does not affect the model and very accurate results can be obtained. For example, an expert who thought the material was good has small deviations from the one who thought "such a medium" in fuzzy logic as small inferences that constitute the difference. With the logical rules that form the basis of a probability table some entries may be eliminated unreasonably. For example, the input such as; "bad material, poor workmanship, the changing form, the appropriate function, the texture of the city preserved in place" was not true logically. Because, if there is a loss in material, form and authenticity of craftsmanship it cannot be expected to have not lost its original function. After all these limitations, the rest can be determined as the total logical output number. 


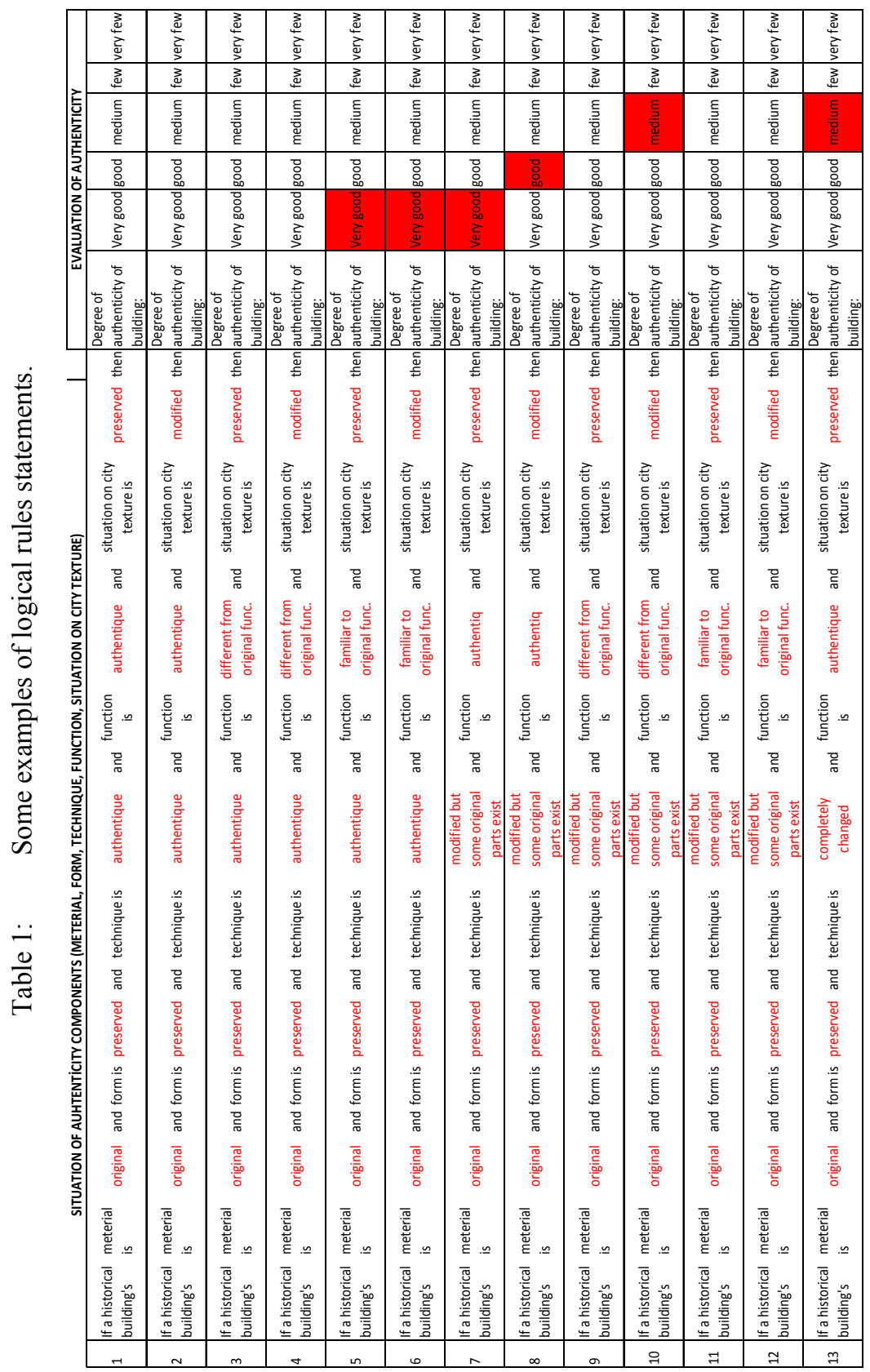




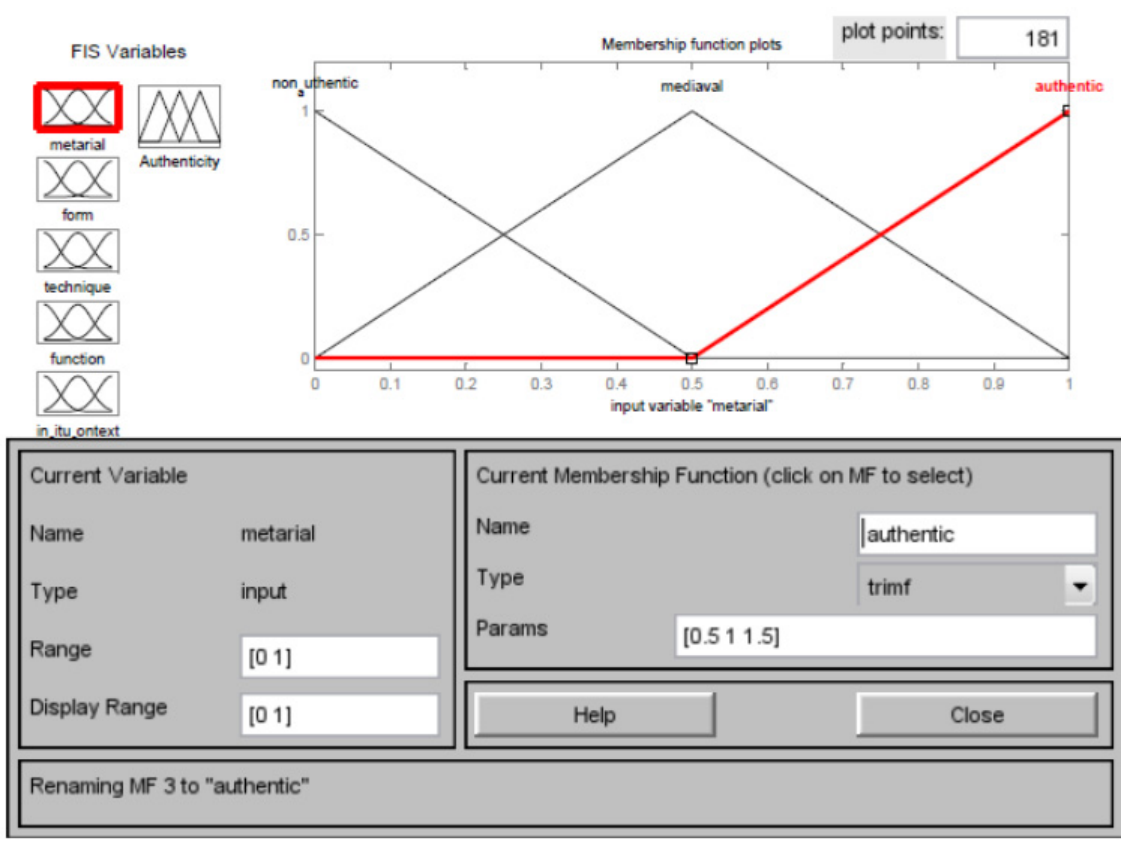

Figure 4: $\quad$ Degree of membership graphic by Matlab [12].

In this way, the degree of membership for other inputs is determined by the same results. By this way, the degree of membership defined between 0 and 1 and the membership degree sub cluster.
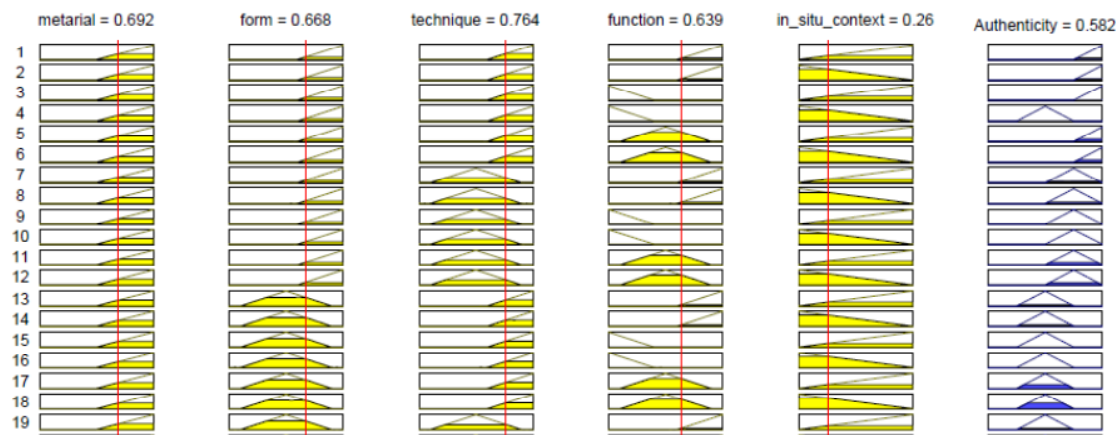

Figure 5: Results of authenticity evaluation using fuzzy logic sets via Matlab2010b [12].

These groups form the fuzzy groups and defined as blur. After this phase to find out clear output we go on with the "defuzzification" operations. According to the type of implementation and required results different defuzzification methods can be selected. Here, the principle of the largest membership, 
Mamdani method, centroid method, weighted average basis, the average of the largest membership, totals, center, the largest space center, the biggest, first or last degree of membership of the methods, such as the "Mamdani Method" is done by selecting the defuzzification process (Figure 2). Such kind of defuzzification and inference with the model established in this way with the computer program MATLAB as the digitized in desired range $(0-100$ or $0-10$, etc.) to obtain results.

The classical logic approach which only defines architectural heritage "authentic or not" trivializes historic buildings. Today, the rating of historical building is a necessity for the authenticity of both the material and form, technical, functional and urban fabric needs to be fully protected. We may clarify that defining a historic building or site with verbal expressions such as less authentic, very authentic, very little authentic expression, we may use numerical expressions of how much is the percentage and have a deeper understanding with fuzzy logic.

\section{Conclusion}

The "authenticity rating model" is a methodology that can be used in the field of architecture conservation. This model provides an "authenticity test" which will help to label the buildings with an authenticity certificate. This certification process will help central decision making for authenticity levels of the buildings. For example, determination of conservation principals for the buildings between $80 \%$ and $90 \%$ authenticity. Also, during the documentation of the existing buildings, the tool will help the expert to take accurate decision-making. This will also provide a shorter process for bureaucracy.

Today, in many parts of the world, it is hard to make decisions because of the solid framework in the field of architectural conservation. This study analyses the potential use of fuzzy logic with a better understanding of the principles of general application problems and the proposed method may contribute to the solution to problems in the field of architectural conservation.

\section{References}

[1] Şen, Z., (2004). Mühendislikte Bulanık (Fuzzy) Mantık ile Modelleme Prensipleri, Su Vakfı Yayınları, İstanbul.

[2] Zadeh, A.L., (1988). Fuzzy Logic, [Online], Available: http://www.geocities.ws/hhvillav/00000053.pdf [20 Haziran 2010].

[3] Şen, Z., (2009). Spatial Modeling Principles in Earth Science, Springer, New York, London.

[4] Ito, N. (1994). Authenticity Inherent in Cultural Heritage in Asia and Japan. In International Council of Monuments and Sites, UNESCO, ICCROM and ICOMOS, Nara Conference. Japan, November 1-6.

[5] International Charter for the Conservation and Restoration of Monuments and Sites (The Venice Charter 1964) IInd International Congress of Architects and Technicians of Historic Monuments, Venice, 1964. 
[6] http://www.kumid.eu/euproject/admin/userfiles/dokumanlar/Dogalmiras.pdf.

[7] The Nara Document on Authenticity, Nara Conference on Authenticity in Relation to the World Heritage Convention, Nara, Japan, 1-6 November 1994. http://www.international.icomos.org/charters.htm.

[8] World Heritage Site, UNESCO, [Online], http://whc.unesco.org/en/list/323 [25 June 2010].

[9] Zadeh, A.L., (1997). Toward a Theory of Fuzzy İnformation Granulation and Its Centrality in Human Reasoning and Fuzzy Logic. Fuzzy Sets and Systems, 90: p.111-127.

[10] http://upload.wikimedia.org/wikipedia/commons/thumb/6/61/Fuzzy_logic temperature_en.svg/1000px-Fuzzy_logic_temperature_en.svg.png.

[11] Nishimura, Y., 2010. Path between Authenticity and Integrity From Nara Document on Authenticity to Historic Urban Landscape, ICOMOS ISC Theory of Conservation, Prague, Czech Republic, 5-9 May 2010.

[12] MatlabR2010b, Licensed to Istanbul Technical University. 